\title{
ARITHMETICAL CONSEQUENCES OF TWO IDENTITIES OF B. GORDON
}

\author{
JOHN A. EWELL \\ Department of Mathematical Sciences \\ Northern Illinois University \\ Dekalb, Illinois 60115 U.S.A.
}

(Received May 28, 1979)

ABSTRACT. From two partition identities of Basil Gordon the author derives two recursive formulas for the sum-of-divisors function. A third application yields an alternate proof of Ramanujan's theorem on the divisibility of certain values of the partition function by the modulus 5 .

KEY WORDS AND PHRASES. Partition identities, sum-of-divisors function, partition function, recurrences.

1980 MATHEMATICS SUBJECT CLASSIFICATION CODES. Primary 10A20; Secondary $10 A 35$.

1. INTRODUCTION.

The partition identities

$$
\begin{aligned}
& \prod_{n=1}^{\infty}\left(1-x^{n}\right)^{3}\left(1-x^{2 n-1}\right)^{2}=\sum_{n=-\infty}^{\infty}(6 n+1) x^{n(3 n+1) / 2}, \\
& \prod_{n=1}^{\infty}\left(1-x^{n}\right)^{3}\left(1-x^{2 n-1}\right)^{-5}=\sum_{n=-\infty}^{\infty}(-1)^{n}(3 n+1) x^{n(3 n+2)}
\end{aligned}
$$


were first proved in [3]. In the present investigation we make three outstanding applications of these identities. As for methods of proof, we follow Euler and Ramanujan. Euler was the first mathematician to use a partition identity to gain important arithmetical knowledge. Specifically, he used his now famous pentagonal-number identity

$$
\prod_{n=1}^{\infty}\left(1-x^{n}\right)=\sum_{n=-\infty}^{\infty}(-1)^{n} x^{n(3 n+1) / 2}
$$

to derive recursive determinations of the sum-of-divisors function and the partition function. Accordingly, let us briefly recall that for each given positive integer $n, \sigma(n)$ denotes the sum of the positive divisors of $n$, and $p(n)$ denotes the number of unrestricted partitions of $n$; conventionally $p(0)=1$. Ramanujan was the first mathematician to observe congruences in certain values of p relative to the moduli 5, 7 and 11. Recently, the author observed that Euler's technique of logarithmic differentiation (which he applied to his identity to derive a recursive formula for $\sigma$ ) can be extended to identities of the type

$$
\prod_{n=1}^{\infty}\left(1-x^{n}\right)^{a}\left(1-x^{2 n-1}\right)^{b}=1+\sum_{n=1}^{\infty} c(a, b ; n) x^{n},
$$

where $(a, b)$ is an arbitrary pair of real numbers, say, and in all of the interesting cases the right-hand series is lacunary having gaps which increase quadratically in size. As a matter of fact, only the special cases: $(a, b)=(1,0),(3,0),(1,1)$, $(1,-2)$ were discussed in the papers [1], [2]. In addition to Euler's identity, the following identities were also used.

$$
\prod_{n=1}^{\infty}\left(1-x^{n}\right)\left(1-x^{2 n-1}\right)=1+2 \sum_{n=1}^{\infty}(-1)^{n} x^{n^{2}},
$$




$$
\begin{aligned}
& \prod_{n=1}^{\infty}\left(1-x^{n}\right)\left(1-x^{2 n-1}\right)^{-2}=\sum_{n=0}^{\infty} x^{n(n+1) / 2}, \\
& \prod_{n=1}^{\infty}\left(1-x^{n}\right)^{3}=\sum_{n=0}^{\infty}(-1)^{n}(2 n+1) x^{n(n+1) / 2}
\end{aligned}
$$

The first two are due to Gauss and the third to Jacobi. The general theorem 1 (stated below) contains all of these results, as well as two new recursive formulas for $\sigma$ corresponding to the special cases: $(a, b)=(3,2),(3,-5)$. Both statement and proof of the theorem are facilitated by the following "natural" DEFINITION. For each positive integer $n$, we express $n$ as $n=2^{b(n)} 0(n)$, where $b(n)$ is a nonnegative integer and $O(n)$ is odd. Then, for each pair $(a, b)$ of real numbers, we define the function $\omega(a, b ;)$ by $\omega(a, b ; n)=a \sigma(n)+b \sigma(0(n))$. (There should be no confusion between the constant $b$ and the functional value $b(n)$ standing for the exponent of the binary part of $n$. )

THEOREM 1. For each given pair $(a, b)$ of real numbers and for each positive integer $\mathrm{m}$,

$$
\begin{aligned}
(a+b)\{\sigma(2 m-1) & \left.+\sum_{1}^{m-1} \sigma(2 m-1-2 k) c(a, b ; 2 k)\right\} \\
& +\sum_{1}^{m-1} w(a, b ; 2 m-1-2 k+1) c(a, b ; 2 k-1) \\
& =-(2 m-1) c(a, b ; 2 m-1)
\end{aligned}
$$

and

$$
\begin{aligned}
w(a, b ; 2 m) & +\sum_{1}^{m-1} w(a, b ; 2 m-2 k) c(a, b ; 2 k) \\
& +(a+b) \sum_{1}^{m} \sigma(2 m-2 k+1) c(a, b ; 2 k-1)
\end{aligned}
$$


Section 2 is dedicated to the proof of theorem 1 and two corollaries. In section 3 we combine Identity (1) with the second of Gauss's Identities to give an alternate proof of Ramamuan's theorem concerning the divisibility of the values $p(5 m+4)$ by 5 .

2. Proof of Theorem 1: Briefly, set $F(x)=1+\sum C(a, b ; n) x^{n}$. Now take the logarithmic derivative of identity (3) and multiply the resulting identity by $x$ to obtain

$$
(a+b) \sum_{1}^{\infty} \sigma(2 m-1) x^{2 m-1}+\sum_{1}^{\infty} \omega(a, b ; 2 m) x^{2 m}=-\frac{x F^{\prime}(x)}{F(x)}
$$

(For deta1ls, see [1] or [2].) Now,

$$
\begin{aligned}
& (a+b)\left\{\sum_{1}^{\infty} \sigma(2 m-1) x^{2 m-1}\right\} F(x) \\
& =(a+b)\left\{\sum_{1}^{\infty} x^{2 m-1}\left(\sigma(2 m-1)+\sum_{k=1}^{m-1} \sigma(2 m-1-2 k) C(a, b ; 2 k)\right)\right. \\
& \left.\quad+\sum_{1}^{\infty} x^{2 m} \sum_{k=1}^{m} \sigma(2 m-2 k+1) C(a, b ; 2 k-1)\right\}
\end{aligned}
$$

And,

$$
\begin{aligned}
& \left\{\sum_{1}^{\infty} \omega(a, b ; 2 m) x^{2 m}\right\} F(x) \\
& =\sum_{1}^{\infty} x^{2 m}\left(\omega(a, b ; 2 m)+\sum_{k=1}^{m-1} \omega(a, b ; 2 m-2 k) c(a, b ; 2 k)\right) \\
& \quad+\sum_{1}^{\infty} 2 m-1 \sum_{k=1}^{m-1} \omega(a, b ; 2 m-1-2 k+1) c(a, b ; 2 k-1)
\end{aligned}
$$


The sum of these two expansions equals $-x F^{\prime}(x)$. Hence, we equate coefficients of odd powers to obtain recurrence (4) and equate coefficients of even powers to obtain recurrence (5).

For the special case $(a, b)=(3,2)$, we rewrite the right side of Identity (1) as $1+\sum_{1}^{\infty}(6 n+1) x^{n(3 n+1) / 2}-\sum_{1}^{\infty}(6 n-1) x^{n(3 n-1) / 2}$ and observe that $\omega(3,2 ; n)=\left(3.2^{b(n)+1}-1\right) \cdot \sigma(0(n))$. Although $n$ odd Implies that $\omega(3,2 ; n)$ $=5 \cdot \sigma(n)$, we do not separate the arguments by parity in order to gain an easy statement of

COROLLARY 1.1: For each positive integer $n$,

$$
\begin{aligned}
& \omega(3,2 ; n)-\sum_{k=1}(6 k-1) \omega\left(3,2 ; n-\left(3 k^{2}-k\right) / 2\right) \\
& +\sum_{k=1}(6 k+1) w\left(3,2 ; n-\left(3 k^{2}+k / 2\right)\right. \\
& = \begin{cases}(6 m-1)\left(3 m^{2}-m\right) / 2, & \text { if } n=\left(3 m^{2}-m\right) / 2, \\
-(6 m+1)\left(3 m^{2}+m\right) / 2, & \text { if } n=\left(3 m^{2}+m\right) / 2, \\
0, \text { otherwise. }\end{cases}
\end{aligned}
$$

As the exponents $3 n^{2}+2 n$ on the right side of identity (2) are easy to separate by parity, we here follow the exact statement of our theorem. But, since $\sigma$ is multiplicative, it suffices to give the specialization of recurrence (4). In order to make the right side of identity (2) have the same form as that of identity (3), we rewrite the right side of identity (2) as $1+\left[(-1)^{n}\left\{(3 n+1) x^{n(3 n+2)}-\right.\right.$ $\left.(3 n-1) x^{n(3 n-2)}\right\}$. The corresponding recurrence is thus put into a form easier to 
apply. (A quick calculation shows that roughly $2 \sqrt{n / 3}$ of the values $\sigma(f)$, $1 \leq f<n$, are needed to compute $\sigma(n)$ for large $n$ odd.)

COROLLARY 1.2: For each positive integer $\mathrm{m}$,

$$
\begin{aligned}
\sigma(2 m-1) & +\sum_{k=1}(6 k+1) \sigma(2 m-1-2 k(6 k+2)) \\
& -\sum_{k=1}(6 k-1) \sigma(2 m-1-2 k(6 k-2)) \\
& +\sum_{k=1}(3 k-1) \omega(3,-5 ; 2 m-1-(2 k-1)(6 k-1)) \\
& -\sum_{k=1}(3 k-2) \omega(3,-5 ; 2 m-1-(2 k-1)(6 k-5)) \\
& =\left\{\begin{array}{l}
j(3 j-2)(3 j-1) / 2, \text { if } 2 m-1=j(3 j-2) \\
-j(3 j+1)(3 j+2) / 2, \text { if } 2 m-1=j(3 j+2) \\
0, \text { otherwise. }
\end{array}\right.
\end{aligned}
$$

3. A Theorem of Ramanujan. The theorem which here concerns us is THEOREM 2. For each nonnegative integer $\mathrm{m}$,

$$
p(5 m+4) \equiv 0 \quad(\bmod 5)
$$

We content ourselves with an outline of how Identity (1) and Gauss's Identity $\Pi\left(1-x^{n}\right)\left(1-x^{2 n-1}\right)^{-2}=\left[x^{n(n+1) / 2}\right.$ combine to yleld a proof of theorem 2 . To this end, we first multiply $x$ and the two identities to obtain

$$
x_{n=1}^{\infty}\left(1-x^{n}\right)^{4}=\sum_{r=-\infty}^{\infty} \sum_{s=0}^{\infty}(6 r+1) x^{k},
$$

where

$$
k=k(r, s)=1+r(3 r+1) / 2+s(s+1) / 2 .
$$


Next, we prove the following

LEMMA: For each pair $(r, s)$ of integers, $s \geq 0$, if 5 divides $k(r, s)$, then 5 also divides each of the integers $(6 r+1)^{2}$ and $2(s+1)^{2}+3 s+1$. Now,

$$
\begin{aligned}
24 k & =(6 r+1)^{2}+12(s+1)^{2}-12 s+11 \\
& \equiv(6 r+1)^{2}+2(s+1)^{2}+3 s+1 \quad(\bmod 5)
\end{aligned}
$$

Hence, the only way to obtain $0(\bmod 5)$ upon adding is to have both $(6 r+1)^{2}$ and $2(s+1)^{2}+3 s+1$ congruent to $0(\bmod 5)$. This proves our 1 emma; and in particular, if 5 divides $k(r, s)$, then 5 divides $6 r+1$.

We now complete the proof of theorem 2 just as in [4, pp. 288, 289] .

REMARKS: In [2] two triangular-number recursive formulas for $\sigma$ are noted. Now, with Euler's formula and that of corollary 1.1, we also have two pentagonalnumber formulas. And, finally in the exponent $k(r, s)=1+r(3 r+1) / 2+s(s+1) / 2$ we have a choice (depending on whether we use the identities of Euler and Jacobi, as did Ramamujan in his original proof, or the identities of Gauss and Gordon), a choic of how we complete squares so as to prove the crucial lemma. On the one hand, the triangular numbers $s(s+1) / 2$ are in the foreground, while on the other hand, the pentagonal numbers $r(3 r+1) / 2$ have center stage.

A reasonable guess is that there is only one recursive formula for $\sigma$ which involves squares. But, the recurrence of corollary 1.2 is curious, to say the least. One glaring point of exception revolves about the exponents $n(3 n+2)$. These can hardly be classified with the common figurate numbers. 


\section{REFERENCES}

1. J. A. Ewell, Recurrences for the sum of divisors, Proc. Amer. Math. Soc. 64 (1977), 214-218.

2. J. A. Ewell, Recursive determination of the sum-of-divisors function, Proc. Amer. Math. Soc. 73 (1979), 169-172.

3. B. Gordon, Some identities in combinatorial analysis, Quart. J. Math. Oxford Ser. (2) 12 (1961), 285-290.

4. G. H. Hardy and E. M. Wright, An introduction to the theory of numbers, 4 th ed., Clarendon Press, Oxford, 1960. 


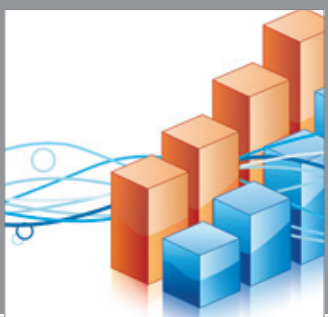

Advances in

Operations Research

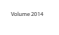

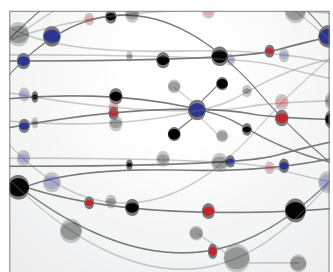

\section{The Scientific} World Journal
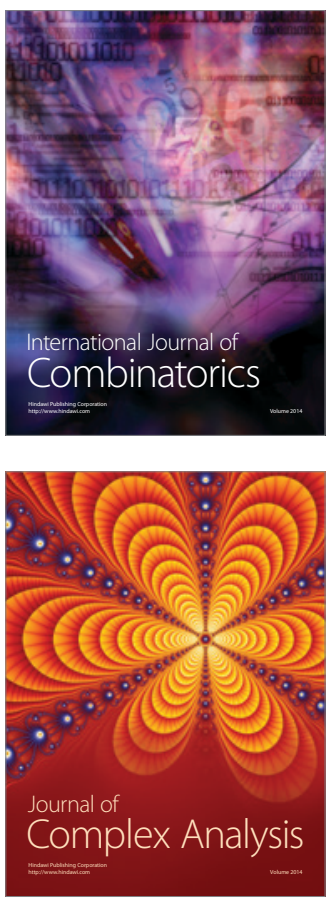

International Journal of

Mathematics and

Mathematical

Sciences
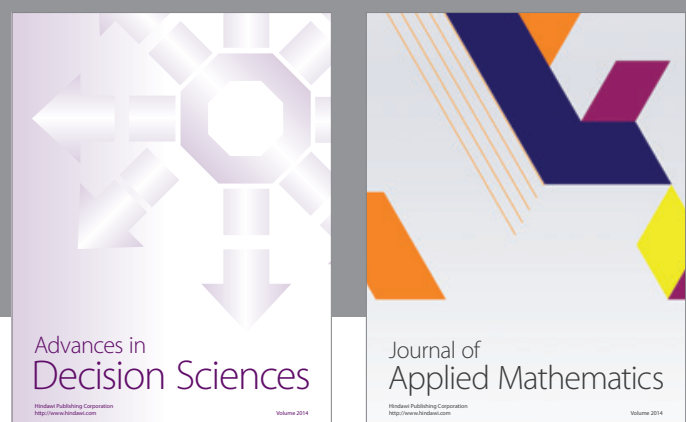

Journal of

Applied Mathematics
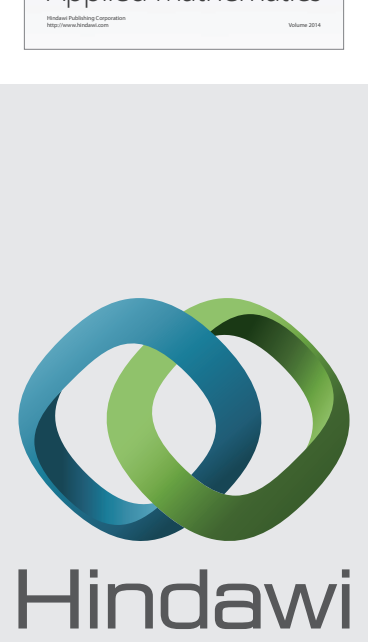

Submit your manuscripts at http://www.hindawi.com
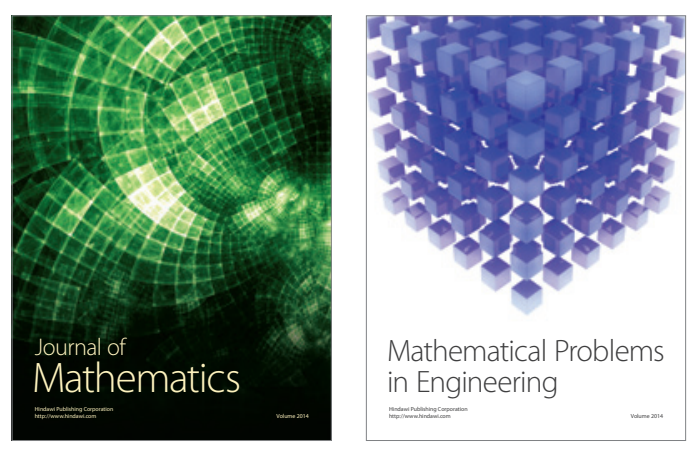

Mathematical Problems in Engineering
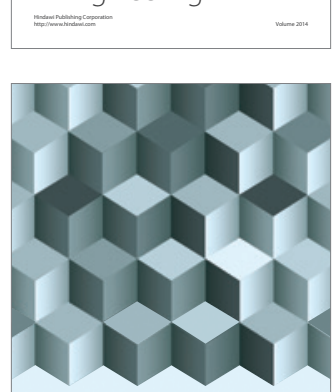

Journal of

Function Spaces
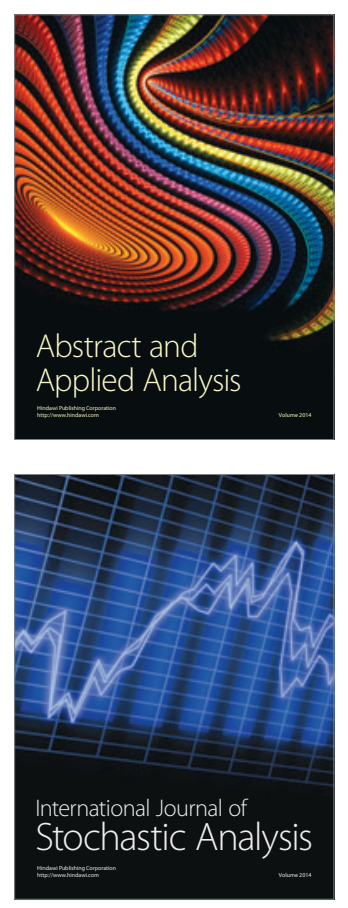

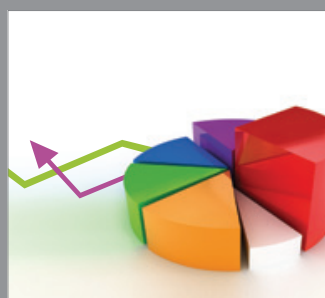

ournal of

Probability and Statistics

Promensencen
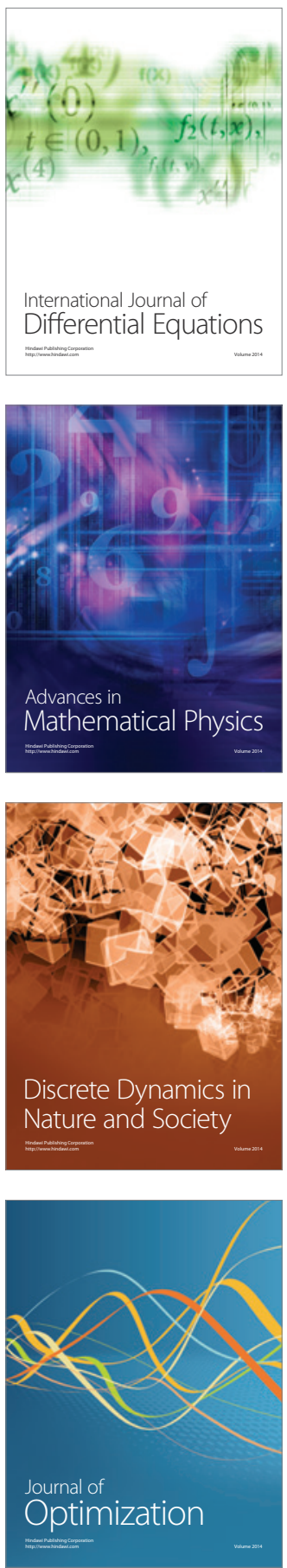